\title{
Universal Taylor series with maximal cluster sets
}

\section{Bernal-González, A. Bonilla, M. C. Calderón-Moreno and J. A. Prado-Bassas}

\begin{abstract}
We link the overconvergence properties of certain Taylor series in the unit disk to the maximality of their cluster sets, so connecting outer wild behavior to inner wild behavior. Specifically, it is proved the existence of a dense linear manifold of holomorphic functions in the disk that are, except for zero, universal Taylor series in the sense of Nestoridis and, simultaneously, have maximal cluster sets along many curves tending to the boundary. Moreover, it is constructed a dense linear manifold of universal Taylor series having, for each boundary point, limit zero along some path which is tangent to the corresponding radius. Finally, it is proved the existence of a closed infinite dimensional manifold of holomorphic functions enjoying the two-fold wild behavior specified at the beginning.
\end{abstract}

\section{Introduction and notation}

In 1996, Nestoridis [46] introduced the following notion, that is a sort of "functional" overconvergence of power series in a disk of the complex plane $\mathbb{C}$.

A holomorphic function $f \in H(\mathbb{D})$ is called a universal Taylor series if, for every compact set $K \in \mathcal{M}(\mathbb{C} \backslash \mathbb{D})$ and every function $h \in A(K)$, there exists a sequence $\left(\lambda_{n}\right) \subset \mathbb{N}_{0}$ such that

$$
s\left(\lambda_{n}, f\right) \longrightarrow h(n \rightarrow \infty) \text { uniformly on } K \text {. }
$$

The sequence $\left(\lambda_{n}\right)$ can be chosen so that $\lambda_{1}<\lambda_{2}<\cdots$. We have used the following notation: $\mathbb{N}_{0}=\mathbb{N} \cup\{0\}$, where $\mathbb{N}$ is the set of positive integers, $\mathbb{D}$ is the open unit disk $\{z \in \mathbb{C}:|z|<1\}, H(G)$ is the space of all holomorphic

2000 Mathematics Subject Classification: Primary: 30B30; Secondary: 30D40, 30E10, 47B38.

Keywords: Maximal cluster set, universal Taylor series, dense linear submanifold, closed linear submanifold, differential operator, curve with non-total oscillation. 
functions in a domain (:= nonempty connected open subset of $\mathbb{C}) G, \mathcal{M}(A)$ is the family of all compact subsets $K \subset A$ such that $\mathbb{C} \backslash K$ is connected $(A \subset \mathbb{C}), A(K)$ is the space of all continuous functions $h: K \rightarrow \mathbb{C}$ that are holomorphic in the interior $K^{0}$ of $K$, and $s(n, f)(z):=\sum_{k=0}^{n} \frac{f^{(k)}(0)}{k !} z^{k}$, the $n$th partial sum of the Taylor expansion of $f$ around the origin. It is known that $H(G)$ is a Fréchet space (:= complete locally convex metrizable topological vector space) under the topology of uniform convergence on compacta in $G$ (so $H(G)$ is a Baire space), while $A(K)$ is a Banach space when endowed with the maximum norm.

In $[46]$ it is proved that the class $U(\mathbb{D})$ of universal Taylor series is residual in $H(\mathbb{D})$, hence nonempty. Hence we can say that, in a topological sense, "most" functions in $H(\mathbb{D})$ possess an extremely wild "outer" boundary behavior, if we consider the overconvergence phenomenon.

It should be observed that Nestoridis' notion is stronger than the one introduced in the seventies by Luh [39] and Chui and Parnes [26], where the compact sets $K$ were only allowed to belong to $\mathcal{M}(\mathbb{C} \backslash \overline{\mathbb{D}}), \bar{A}$ denoting the closure of a subset $A \subset \mathbb{C}$. Since Nestoridis' result, much effort has been done in the research of the universal Taylor series. Some of the related statements are mentioned along this paper; for others, see e.g. [2] and the references contained in it.

Now, let us adopt for a moment a different point of view, namely, that of the "inner" boundary behavior. Let $G$ be a domain, $f \in H(G)$ and $\gamma:[0,1) \rightarrow \mathbb{C}$ be a curve in $G$ tending to the boundary $\partial G$ (that is, $\gamma$ is continuous, $\gamma([0,1)) \subset G$ and, given a compact set $K \subset G$, there is $t_{0} \in(0,1)$ such that $\gamma\left(\left(t_{0}, 1\right)\right) \cap K=\emptyset$; by abuse of notation, we will sometimes identify $\gamma$ with its image $\gamma([0,1)))$, then the cluster set of $f$ along $\gamma$ is defined as

$C_{\gamma}(f)=\left\{w \in \mathbb{C}_{\infty}\right.$ : there exists $\left\{t_{n}\right\}_{1}^{\infty} \subset[0,1)$ with $t_{n} \rightarrow 1$ and $\left.f\left(\gamma\left(t_{n}\right)\right) \rightarrow w\right\}$, where $\mathbb{C}_{\infty}$ denotes the extended complex plane $\mathbb{C} \cup\{\infty\}$ (see [25] and [47] for an account of results about cluster sets). A cluster set is said to be maximal whenever it equals $\mathbb{C}_{\infty}$. In 1933, Kierst and Szpilrajn [36] proved that there is a residual subset of functions in $H(\mathbb{D})$ having maximal cluster set at each point of $\mathbb{T}:=\partial \mathbb{D}$. Even more, in $[36$, Section 4$]$ it is stated that there is a residual subset of functions in $H(\mathbb{D})$ having maximal cluster set along every curve $\gamma \in \Gamma_{0}(\mathbb{D})$. We have denoted, for every domain $G$,

$$
\begin{aligned}
& \Gamma_{0}(G):=\{\text { curves } \gamma \subset G \text { tending to the boundary such that there is a } \\
& \text { connected set } A=A(\gamma) \subset G \text { with } \bar{A} \cap \partial G \neq \emptyset=A \cap \gamma\} \text {. }
\end{aligned}
$$

(In [36] the set $A$ was a segment, but the proof would be the same for our more general family; note also that the class $\Gamma_{0}(G)$ contains strictly 
the class $\Gamma(G)$ of curves $\gamma \subset G$ tending to the boundary with non-total oscillation, that is, such that $(\partial G) \backslash \bar{\gamma} \neq \emptyset$; the class $\Gamma(G)$ is considered in [12] and [14], and a glance at the proofs reveals that the results in these two papers hold for $\Gamma_{0}(G)$ instead of $\Gamma(G)$.) (See also [15, Theorem 2.1] for an extension of the Kierst-Szpilrajn statement where certain holomorphic operators participate.) Since the intersection of two residual subsets is also residual, we can assert, as a consequence of the results by Kierst-Szpilrajn and Nestoridis, that "most" functions in $H(\mathbb{D})$ exhibit, simultaneously, an extremely wild "inner" and "outer" boundary behavior; in other words, this two-fold property is "topologically generic".

In order to put our research into an appropriate setting, we recall some concepts, which have been matter of intensive study in the recent years by several mathematicians. If $A$ is a subset of a topological vector space $X$, then $A$ is called lineable (dense-lineable, spaceable, resp.) provided that there exists an infinite-dimensional (a dense, an infinite-dimensional closed, resp.) linear submanifold $\mathcal{M} \subset X$ such that $\mathcal{M} \backslash\{0\} \subset A$. Following [6], a property on $X$ is said to be algebraically generic if the set $A$ of vectors in $X$ satisfying such property is dense-lineable. Lineability has been widely studied in [4], [5], [1], [30], [29] and [33], among others. The matter has interest whenever $A$ is not a linear space. For instance, the existence of dense linear manifolds of hypercyclic vectors (i.e. vectors with dense orbits) for general operators was solved in [22] for the complex case, and in [18] for the real case.

Obviously, neither the set $U(\mathbb{D})$ nor the set

$$
\operatorname{MCS}\left(\Gamma_{0}(\mathbb{D})\right):=\left\{f \in H(\mathbb{D}): C_{\gamma}(f)=\mathbb{C}_{\infty} \text { for all } \gamma \in \Gamma_{0}(\mathbb{D})\right\}
$$

are linear spaces. But, according to the terminology of the last paragraph, one could wonder if the property of wild inner-outer boundary behavior mentioned before is algebraically generic in $H(\mathbb{D})$. If we consider each property independently of the other, the answer is "yes". To be more precise, in [14, Theorem 2.1] it is proved that there is a dense linear submanifold $\mathcal{D}$ in $H(\mathbb{D})$ with $\mathcal{D} \backslash\{0\} \subset M C S\left(\Gamma_{0}(\mathbb{D})\right)$; and according to [6, Remark (3) on page 176], the hypercyclicity methods developed in [6] lead to a similar conclusion for $U(\mathbb{D})$. But this does not imply that both sets share such a common submanifold $\mathcal{D}$. Nevertheless, we will prove that the answer is also affirmative: Belonging to $U(\mathbb{D}) \cap M C S\left(\Gamma_{0}(\mathbb{D})\right)$ is an algebraically generic property. In fact, this statement will be formulated in a much more general way (see Theorem 3.1), involving sequences of holomorphic operators and infinite matrices with adequate properties.

Observe that our result complements one due to Melas and Nestoridis [42] asserting that, for every $f \in U(\mathbb{D})$ and every boundary point $\zeta \in \mathbb{T}$, the 
limit $\lim _{z \rightarrow \zeta, z \in \mathbb{D}} f(z)$ does not exist in the complex plane. It follows that $\mathbb{T}$ is a natural boundary for every universal Taylor series, which provides an affirmative answer to a conjecture of J.-P. Kahane [35]. In the same line of "bad" behavior, Armitage, Costakis and Bayart have recently proved (see $[2$, Theorem 1.1] and [8, Theorem 3.2]) that if $f \in U(\mathbb{D})$ then there exists a residual subset $M$ of $\mathbb{T}$, such that the set

$$
\left\{f^{(n)}(r \zeta): 0<r<1\right\}
$$

is unbounded for every $\zeta \in M$ and every $n \in \mathbb{N}_{0}$. We improve this statement (see Theorem 4.1) by replacing the derivatives by linear differential operators.

In the opposite direction, Costakis and Armitage (see [27] and [2]) have also showed that, given a subset $E \subset \mathbb{T}$ of first category in $\mathbb{T}$, there exist universal Taylor series with radial limit at every point of $E$. We will also complement this result by constructing a dense linear manifold of universal Taylor series having, for each boundary point, limit zero along some path which is tangent to the radius joining the origin with the point, see Theorem 4.2.

Finally, we deal with the other mentioned kind of large algebraic structure: the spaceability. Probably, the starting point of spaceability was the result of Read [50] about the existence of hypercyclic operators on $l^{1}$ such that every non-null vector has a dense orbit. A systematic study of the existence of closed infinite dimensional subspaces with some universal behavior was initiated by Montes and the first author in [17]. A very general sufficient condition was obtained in [43]. This was refined in [38], and an optimal result in the context of Banach spaces was obtained in [31]. Using different techniques based on the study of spaces of operators with the strong operator topology, Chan [23] obtained in an easy way the original result of [43] for Hilbert spaces. This was latter generalized to Banach spaces in [24], [44] and [41]. Extensions to the context of Fréchet spaces can be found in [20], [11], [49] and [3]. Concerning our research in this paper, Bayart [7, Theorem 2] was able to prove the spaceability of $U(\mathbb{D})$ in $H(\mathbb{D})$. We complete his result as well as our Theorem 3.1 by proving the spaceability of $U(\mathbb{D}) \cap M C S\left(\Gamma_{0}(\mathbb{D})\right)$ in $H(\mathbb{D})$ (see Theorem 5.2).

\section{Further terminology and preliminary results}

Firstly, let us recall a dynamic concept (see [32] and [37] for excellent surveys). Assume that $L_{n}: X \rightarrow Y(n \in \mathbb{N})$ is a sequence of continuous mappings between two topological spaces $X, Y$. Then the sequence $\left(L_{n}\right)$ is called universal provided that there exists an element $x_{0} \in X$-called 
universal for $\left(L_{n}\right)$ - whose orbit $\left\{L_{n} x_{0}: n \in \mathbb{N}\right\}$ under $\left(L_{n}\right)$ is dense in $Y$. If the set $\mathcal{U}\left(\left(L_{n}\right)\right)$ of universal elements for $\left(L_{n}\right)$ is not only nonempty but also dense in $X$, then $\left(L_{n}\right)$ is called densely universal. If $X$ is a Baire space and $Y$ is second-countable, then $\left(L_{n}\right)$ is densely universal if and only if $\mathcal{U}\left(\left(L_{n}\right)\right)$ is residual. The word "universal" is usually replaced by "hypercyclic" when $X, Y$ are topological vector spaces and the mappings $L_{n}$ are linear.

Let $\mathcal{A}=\left[\alpha_{n \nu}\right]_{n, \nu=0}^{\infty}$ be an infinite matrix with complex entries. In order to save some notation, we introduce the following definition. We say that $\mathcal{A}$ is a $C$-matrix if it satisfies the next three conditions:

(i) For all $n \in \mathbb{N}_{0}, \lim _{\nu \rightarrow \infty}\left|\alpha_{n \nu}\right|^{1 / \nu}=0$.

(ii) For all $\nu \in \mathbb{N}_{0}, \lim _{n \rightarrow \infty} \alpha_{n \nu}=0$.

(iii) The limit $\lim _{n \rightarrow \infty} \sum_{\nu=0}^{\infty} \alpha_{n \nu}$ exists and is finite and non-zero.

Note that, due to (i), we have that each series $\sum_{\nu=0}^{\infty} \alpha_{n \nu}\left(n \in \mathbb{N}_{0}\right)$ occurring in (iii) converges. In fact (i) tells us that each row of $\mathcal{A}$ is the sequence of the Taylor coefficients (at the origin) of some entire function $\Phi_{n}$, while (iii) tells us that there exists $\lim _{n \rightarrow \infty} \Phi_{n}(1) \in \mathbb{C} \backslash\{0\}$.

Assume that $\mathcal{A}$ is a $C$-matrix and that $f \in H(\mathbb{D})$. According to $[13$, Lemma 2.3] (with $R_{0}=+\infty$ ), the sequence

$$
\sigma_{\mathcal{A}}(n, f)(z):=\sum_{\nu=0}^{\infty} \alpha_{n \nu} s(\nu, f)(z)
$$

of $\mathcal{A}$-transforms of $f$ is well defined on $\{|z| \geq 1\}$ and, in fact, $\sigma_{\mathcal{A}}(n, f) \in$ $A(K)$ for each compact set $K \subset\{|z| \geq 1\}$. We say that a function $f \in H(\mathbb{D})$ is $\mathcal{A}$-universal if, for every compact set $K \in \mathcal{M}(\mathbb{C} \backslash \mathbb{D})$ and every function $h \in A(K)$, there exists a sequence $\left(\lambda_{n}\right) \subset \mathbb{N}_{0}$ such that

$$
\sigma_{\mathcal{A}}\left(\lambda_{n}, f\right) \longrightarrow h(n \rightarrow \infty) \text { uniformly on } K \text {. }
$$

Therefore $f \in U(\mathbb{D})$ is the same as $f$ is $I$-universal, $I$ being the (infinite) identity matrix. The study of the universal behavior of matrix transforms of holomorphic functions has been dealt with by several authors (see for instance [32, Sect. 4e] and [42]).

The following auxiliary result can be found in [13, Theorem 2.2 and Final Remark 4.(5)] (take $R_{0}=+\infty$ there).

Theorem 2.1. Let $\mathcal{A}$ be a $C$-matrix and $\{m(1)<m(2)<m(3)<\cdots\} \subset \mathbb{N}$. Let us denote by $U(\mathbb{D}, \mathcal{A},(m(n)))$ the set of functions $f \in H(\mathbb{D})$ such that, for every compact set $K \in \mathcal{M}(\mathbb{C} \backslash \mathbb{D})$ and every function $h \in A(K)$, there exists a sequence $\left(\lambda_{n}\right) \subset \mathbb{N}_{0}$ satisfying

$$
\sigma_{\mathcal{A}}\left(m\left(\lambda_{n}\right), f\right) \longrightarrow h(n \rightarrow \infty) \text { uniformly on } K \text {. }
$$

Then $U(\mathbb{D}, \mathcal{A},(m(n)))$ is residual in $H(\mathbb{D})$. 
Note that $U(\mathbb{D}, \mathcal{A},(n))$ is the set of $\mathcal{A}$-universal functions. Hence, in particular, this set is residual (so dense) if $\mathcal{A}$ is a $C$-matrix. Note also that $U(\mathbb{D}, I,(n))=U(\mathbb{D})$.

We now reproduce a useful statement about "induced universality" due to Herzog [34]. Recall that a metric space $(X, d)$ is said to be a Polish space if it is separable and complete (so it is Baire and second-countable). Moreover, by Alexandroff's theorem (see [48, pp. 47-48]), a subset $A$ of a complete metric space is completely metrizable if and only if $A$ is a $G_{\delta}$ subset.

Theorem 2.2. Assume that $\left(X, d_{X}\right)$ is a Polish space and $\left(Y, d_{Y}\right)$ is a separable metric space. Assume also that $\left\{A_{k}\right\}_{k=1}^{\infty}$ is a sequence of open subsets of $X$ such that $A:=\bigcap_{k=1}^{\infty} A_{k}$ is nonempty. Let $L_{n}: X \rightarrow Y(n \in \mathbb{N})$ be a densely universal sequence of mappings. If

$$
\lim _{k \rightarrow \infty} \sup _{n \in \mathbb{N}} \inf _{z \in A}\left\{d_{X}\left(a_{k}, z\right)+d_{Y}\left(L_{n} a_{k}, L_{n} z\right)\right\}=0
$$

for every sequence $\left\{a_{k}\right\}_{k=1}^{\infty}$ with $a_{k} \in A_{k}(k \in \mathbb{N})$, then the sequence of restrictions $\left.L_{n}\right|_{A}: A \rightarrow Y(n \in \mathbb{N})$ is also densely universal. Hence $\mathcal{U}\left(\left(\left.L_{n}\right|_{A}\right)\right)$ is residual in $A$.

Next, we present a concept that was introduced by the authors in [12]. If $G \subset \mathbb{C}$ is a domain, then we say that an operator (= linear continuous self-map) $T: H(G) \rightarrow H(G)$ is internally controlled if given any $\varepsilon>0$ and a pair of compact sets $F, L \subset G$ with $F \subset L^{0}$, there exists $\delta=\delta(\varepsilon, F, L)>0$ such that

$$
\left[f \in H(G) \text { and }\|f\|_{L}<\delta\right] \text { implies }\|T f\|_{F}<\varepsilon \text {. }
$$

We have denoted by $\|g\|_{A}$ the quantity $\sup _{z \in A}|g(z)|$, for any function $g$ : $A \rightarrow \mathbb{C}$.

For instance, let $D: f \in H(G) \mapsto f^{\prime} \in H(G)$ be the derivative operator, and assume that $\Phi(z):=\sum_{n=0}^{\infty} a_{n} z^{n}$ is an entire function of subexponential type, that is, given $\varepsilon>0$ there is a constant $A \in(0,+\infty)$ such that $|\Phi(z)| \leq$ $A \exp (\varepsilon|z|)$ for all $z \in \mathbb{C}$. Then its associated (in general, infinite order) differential operator

$$
\Phi(D)=\sum_{n=0}^{\infty} a_{n} D^{n}: H(G) \rightarrow H(G)
$$

defined as $\Phi(D) f=\sum_{n=0}^{\infty} a_{n} f^{(n)}$ is internally controlled on $H(G)$. To see this it is enough to apply the Cauchy integral formula for derivatives together with the fact that $\Phi$ is of subexponential type if and only if $\lim _{n \rightarrow \infty}\left(n\left|a_{n}\right|^{1 / n}\right)=0$ (see for instance [21]). 
As for a negative example, it is not difficult to prove by using Runge's approximation theorem (see [28]) that if $\varphi: G \rightarrow G$ is any analytic self-map which is not the identity, then the corresponding composition operator

$$
C_{\varphi}: f \in H(G) \mapsto f \circ \varphi \in H(G)
$$

is not internally controlled.

Finally, another property to be imposed in Section 3 on an operator $T$ on $H(\mathbb{D})$ will be that $\operatorname{ran} T(:=T(H(\mathbb{D})))$ contains the constant functions. Of course, this is equivalent to $1 \in \operatorname{ran} T$. For instance, every non-zero differential operator $\Phi(D)$ as above satisfies this condition. The operator $f \mapsto I \cdot f$, where $I(z):=z$, does not satisfy it.

\section{Dense linear manifolds of wild functions}

We are now ready to state our main result.

Theorem 3.1. Let $\mathcal{A}$ be a $C$-matrix and $T_{n}: H(\mathbb{D}) \rightarrow H(\mathbb{D})(n \in \mathbb{N})$ be a sequence of operators satisfying the following conditions:

(a) Each $T_{n}$ is internally controlled.

(b) For each $n \in \mathbb{N}$, ran $T_{n}$ contains the constants.

Then there exists a dense linear manifold $\mathcal{D}$ in $H(\mathbb{D})$ satisfying the following properties:

(A) For every function $f \in \mathcal{D} \backslash\{0\}$ and every $n \in \mathbb{N}$, we have that $T_{n} f \in$ $\operatorname{MCS}\left(\Gamma_{0}(\mathbb{D})\right)$.

(B) Every function $f \in \mathcal{D} \backslash\{0\}$ is an $\mathcal{A}$-universal Taylor series.

Proof. We start by choosing a number of families of sets or functions. We select a dense sequence $\left(q_{k}\right)_{k \geq 1}$ in $\mathbb{C}$, as well as a dense sequence $\left(P_{k}\right)_{k \geq 1}$ in $H(\mathbb{D})$; for instance, $\left(q_{k}\right)_{k \geq 1}$ and $\left(P_{k}\right)_{k \geq 1}$ may be, respectively, an enumeration of the set $\mathbb{Q}+i \mathbb{Q}$ of complex rational numbers and an enumeration of polynomials whose coefficients have real and imaginary parts in $\mathbb{Q}+i \mathbb{Q}$. By $\left(K_{k}\right)_{k \geq 1}$ we denote a sequence of compact sets belonging to $\mathcal{M}(\mathbb{C} \backslash \mathbb{D})$ such that every $K \in \mathcal{M}(\mathbb{C} \backslash \mathbb{D})$ is contained in some $K_{k}$ depending on $K$ (see [46]). Fix any pair of sequences $\left(r_{j}\right),\left(s_{j}\right)$ with $0<r_{1}<s_{1}<r_{2}<s_{2}<$ $\cdots<r_{j}<s_{j}<\cdots \rightarrow 1$, and consider the spiral-like compact sets

$$
S_{j}=\left\{\left(r_{j}+\frac{\left(s_{j}-r_{j}\right) \theta}{4 \pi}\right) \exp (i \theta): \theta \in[0,4 \pi]\right\} \quad(j \in \mathbb{N}) .
$$


Observe that the sets $S_{j}$ are contained in $\mathbb{D}$ and are pairwise disjoint. Due to this, one can find $\alpha_{j}>0(j \geq 1)$ such that the sets

$$
\widetilde{S}_{j}:=\left\{z \in \mathbb{C}: \text { there exists } w \in S_{j} \text { with }|z-w| \leq \alpha_{j}\right\}
$$

are still compact, contained in $\mathbb{D}$ and pairwise disjoint. Note also that $S_{j} \subset \widetilde{S}_{j}^{0}$.

Let us denote by $\bar{B}(a, r)$ the closed ball $\{z:|z-a| \leq r\}$ with center $a$ and radius $r>0$. For each $k$, we set $J(k):=\min \left\{j \in \mathbb{N}: r_{j}-\alpha_{j}>k /(k+1)\right\}$ (so $\widetilde{S}_{j} \cap \bar{B}(0, k /(k+1))=\emptyset$ if and only if $\left.j \geq J(k)\right)$ and

$$
F_{k}:=\bar{B}\left(0, \frac{k}{k+1}\right) \cup \bigcup_{j=J(k)}^{\infty} \widetilde{S}_{j} .
$$

Choose also countably many disjoint strictly increasing sequences of positive integers $(p(n, k, j))_{j \geq 1}(n, k \in \mathbb{N})$ whose union is $\mathbb{N}$; note that $p(n, k, j) \geq j$ for all $n, k, j$. By the hypothesis on the range, for each $n \in \mathbb{N}$ there exists a function $h_{n} \in H(\mathbb{D})$ with

$$
T_{n} h_{n}=1 \text {. }
$$

Moreover, by the hypothesis on the internal control, one can find, for every $n \in \mathbb{N}$, every $j \in \mathbb{N}$ and $\varepsilon>0$, a number $\delta=\delta(\varepsilon, n, j)>0$ such that

$$
\left[f \in H(\mathbb{D}) \text { and }\|f\|_{\widetilde{S}_{j}}<\delta\right] \quad \Longrightarrow\left\|T_{n} f\right\|_{S_{j}}<\varepsilon \text {. }
$$

In order to save some notation, we set $N_{k}^{2}:=\left\{(n, l) \in \mathbb{N}^{2}: p(n, k, l) \geq J(k)\right\}$ and $N_{k}^{3}:=\left\{\left(n, k^{\prime}, l\right) \in \mathbb{N}^{3}: k^{\prime} \neq k\right.$ and $\left.p\left(n, k^{\prime}, l\right) \geq J(k)\right\}(k \in \mathbb{N})$. With all this in mind, we define, for each $k \in \mathbb{N}$, the set

$$
\begin{aligned}
& B_{k}:=\{f \in H(\mathbb{D}):\left\|f-P_{k}\right\|_{\bar{B}\left(0, \frac{k}{k+1}\right)}<1 / k, \\
&\left\|f-q_{l} h_{n}\right\|_{\widetilde{S}_{p(n, k, l)}}<\delta\left(\frac{1}{l}, n, p(n, k, l)\right) \text { for all }(n, l) \in N_{k}^{2}, \\
&\text { and } \left.\|f\|_{\widetilde{S}_{p\left(n, k^{\prime}, l\right)}}<\delta\left(\frac{1}{l}, n, p\left(n, k^{\prime}, l\right)\right) \text { for all }\left(n, k^{\prime}, l\right) \in N_{k}^{3}\right\} .
\end{aligned}
$$

Fix $k \in \mathbb{N}$. The set $B_{k}$ is, evidently, a countable intersection of open subsets of $H(\mathbb{D})$, so a $G_{\delta}$ subset. What is less evident is that $B_{k} \neq \emptyset$. Nevertheless, this is true by the Nersesjan tangential approximation theorem (see [45] and [28]). Indeed, if we denote by $\mathbb{D}^{\omega}$ the one-point $(=: \omega)$ compactification of $\mathbb{D}$, then we have:

- $F_{k}$ is a closed proper subset of $\mathbb{D}$.

- $\mathbb{D}^{\omega} \backslash F_{k}$ is connected, because neither $\bar{B}(0, k /(k+1))$ nor the spirals have "holes". 
- $\mathbb{D}^{\omega} \backslash F_{k}$ is locally connected at $\omega$, because two points near $\infty$ can be connected by an arc that is close to $\omega$ and that does not intersect any $\widetilde{S}_{j}$.

- $F_{k}$ does not possess "large islands" near $\infty$, that is, for every compact set $K \subset \mathbb{D}$ there is a neighborhood $W$ of $\omega$ in $\mathbb{D}^{\omega}$ such that no component of $F_{k}^{0}$ intersects both $K$ and $W$. This is so because the components of $F_{k}$ are compact and go towards $\mathbb{T}$.

Furthermore, the function $g_{k}: F_{k} \rightarrow \mathbb{C}$ defined as

$$
g_{k}(z)= \begin{cases}P_{k}(z) & \text { if } z \in \bar{B}\left(0, \frac{k}{k+1}\right) \\ q_{l} h_{n}(z) & \text { if } z \in \widetilde{S}_{p(n, k, l)} \text { and }(n, l) \in N_{k}^{2} \\ 0 & \text { if } z \in \widetilde{S}_{p\left(n, k^{\prime}, l\right)} \text { and }\left(n, k^{\prime}, l\right) \in N_{k}^{3}\end{cases}
$$

satisfies $g_{k} \in A\left(F_{k}\right)$, and the function $\varepsilon_{k}: F_{k} \rightarrow(0,+\infty)$ given by

$$
\varepsilon_{k}(z)= \begin{cases}\frac{1}{k} & \text { if } z \in \bar{B}\left(0, \frac{k}{k+1}\right) \\ \delta\left(\frac{1}{l}, n, p(n, k, l)\right) & \text { if } z \in \widetilde{S}_{p(n, k, l)} \text { and }(n, l) \in N_{k}^{2} \\ \delta\left(\frac{1}{l}, n, p\left(n, k^{\prime}, l\right)\right) & \text { if } z \in \widetilde{S}_{p\left(n, k^{\prime}, l\right)} \text { and }\left(n, k^{\prime}, l\right) \in N_{k}^{3}\end{cases}
$$

is continuous. Hence, by Nersesjan's theorem, the set $\{f \in H(\mathbb{D}): \mid f(z)-$ $g_{k}(z) \mid<\varepsilon_{k}(z)$ for all $\left.z \in F_{k}\right\}$ is nonempty. But this set equals our $B_{k}$. Thus, $B_{k}$ is a nonempty $G_{\delta}$ subset of $H(\mathbb{D})$.

Incidentally, we note that thanks to (1) and (2) one obtains

$$
\left|\left(T_{n} f\right)(z)-q_{l}\right|<\frac{1}{l} \text { for all } z \in S_{p(n, k, l)} \text { and all }(n, l) \in N_{k}^{2}
$$

and

$$
\left|\left(T_{n} f\right)(z)\right|<\frac{1}{l} \text { for all } z \in S_{p\left(n, k^{\prime}, l\right)} \text { and all }\left(n, k^{\prime}, l\right) \in N_{k}^{3} .
$$

Recall that $k$ has already been fixed. Our next task is to prove the following claim: For every strictly increasing sequence $(m(n)) \subset \mathbb{N}$ and every $K \in \mathcal{M}(\mathbb{C} \backslash \mathbb{D})$, the set $B_{k} \cap U(\mathbb{D}, \mathcal{A},(m(n)), K)$ residual in $B_{k}$. We have denoted by $U(\mathbb{D}, \mathcal{A},(m(n)), K)$ the same family of functions described in Theorem 2.1, with the sole exception that the approximation property is restricted to $K$. Thus $U(\mathbb{D}, \mathcal{A},(m(n)))=\bigcap_{K \in \mathcal{M}(\mathbb{C} \backslash \mathbb{D})} U(\mathbb{D}, \mathcal{A},(m(n)), K)$. We proceed similarly to the proof of Theorem 1.2 in [27]. By using the exhaustion property of $\left(K_{k}\right)_{k \geq 1}$ together with the Mergelyan approximation theorem (see [28]), we derive that

$$
U(\mathbb{D}, \mathcal{A},(m(n)))=\bigcap_{k=1}^{\infty} U\left(\mathbb{D}, \mathcal{A},(m(n)), K_{k}\right) .
$$


Consequently, if we were able to prove the property stated at the beginning of this paragraph, then we would arrive at this conclusion:

$$
\text { Each set } B_{k} \cap U(\mathbb{D}, \mathcal{A},(m(n))) \text { is residual in } B_{k} \text {. }
$$

Let us fix a sequence $(m(n))$ and a compact set $K$ as above. It is possible to select a sequence $\left(t_{j}\right)$ of numbers such that

$$
\frac{k}{k+1}<t_{j} \uparrow 1 \quad \text { and } \quad\left\{|z|=t_{j}\right\} \cap \widetilde{S}_{l}=\emptyset \quad \text { for all } \quad j, l \in \mathbb{N} .
$$

The space $X:=H(\mathbb{D})$ is a Polish space under the distance

$$
d_{X}(f, g):=\sum_{j=1}^{\infty} \frac{\|f-g\|_{\bar{B}\left(0, t_{j}\right)}}{1+\|f-g\|_{\bar{B}\left(0, t_{j}\right)}},
$$

which induces the topology of locally uniform convergence, while the distance $d_{Y}(f, g):=\|f-g\|_{K}$ derived from the supremum norm makes the space $Y:=A(K)$ a metric space. This space is also separable by, for instance, Mergelyan's theorem. On the other hand, we have that

$$
B_{k} \cap U(\mathbb{D}, \mathcal{A},(m(n)), K)=\mathcal{U}\left(\left.L_{n}\right|_{A}\right),
$$

where $A:=B_{k}$ and $\left(L_{n}\right)$ is the sequence of continuous linear mappings given by

$$
L_{n}: f \in H(\mathbb{D}) \mapsto \sigma_{\mathcal{A}}(m(n), f) \in A(K) .
$$

Moreover, $A$ can be written as $A=\bigcap_{j=1}^{\infty} A_{j}$, where

$$
A_{j}:=\left\{f \in H(\mathbb{D}):\left|f(z)-g_{k}(z)\right|<\varepsilon_{k}(z) \text { for all } z \in F_{k} \cap \bar{B}\left(0, t_{j}\right)\right\} .
$$

Now, fix $j \in \mathbb{N}$ and $f \in A_{j}$. We are going to show the existence of a sequence $\left(\varphi_{l}\right)_{l \geq 1} \subset B_{k}$ such that

$$
\lim _{l \rightarrow \infty}\left\|f-\varphi_{l}\right\|_{\bar{B}\left(0, t_{j}\right)}=0 .
$$

For this, it is enough to fix an $\varepsilon>0$ and to find a function $\varphi \in B_{k}$ with $|f(z)-\varphi(z)|<\varepsilon$ for all $z \in \bar{B}\left(0, t_{j}\right)$. It is clear that, along with $F_{k}$, the relative closed subset $F_{k} \cup \bar{B}\left(0, t_{j}\right)$ also fulfils the above four topological properties in order to apply Nersesjan's theorem. By the choice of the radii $t_{j}$, the sets $\bar{B}\left(0, t_{j}\right)$ and $F_{k} \backslash \bar{B}\left(0, t_{j}\right)$ can be separated by open sets, so the functions $\Phi: F_{k} \cup \bar{B}\left(0, t_{j}\right) \rightarrow \mathbb{C}$ and $\varepsilon^{*}: F_{k} \cup \bar{B}\left(0, t_{j}\right) \rightarrow(0,+\infty)$ given respectively by

$$
\Phi(z)= \begin{cases}g_{k}(z) & \text { if } z \in F_{k} \backslash \bar{B}\left(0, t_{j}\right) \\ f(z) & \text { if } z \in \bar{B}\left(0, t_{j}\right)\end{cases}
$$


and

$\varepsilon^{*}(z)=\left\{\begin{array}{l}\varepsilon_{k}(z) \\ \min \left\{\varepsilon, \min _{w \in F_{k} \cap \bar{B}\left(0, t_{j}\right)}\left\{\varepsilon_{k}(w)-\left|f(w)-g_{k}(w)\right|\right\}\right\} \quad \text { if } z \in \bar{B}\left(0, F_{j} \backslash \bar{B}\left(0, t_{j}\right)\right.\end{array}\right.$

are well defined and continuous. In addition, $\Phi \in A\left(F_{k} \cup \bar{B}\left(0, t_{j}\right)\right)$. Then there is a function $\varphi \in H(\mathbb{D})$ such that $|\Phi(z)-\varphi(z)|<\varepsilon^{*}(z)$ for all $z \in$ $F_{k} \cup \bar{B}\left(0, t_{j}\right)$. This function $\varphi$ solves our problem, so (6) is true.

Since $K$ is bounded, there is $R_{0} \in(1,+\infty)$ for which $K \subset \bar{B}\left(0, R_{0}\right)$. From the Cauchy inequalities, we get

$$
\left|\frac{f^{(\nu)}(0)-\varphi_{l}^{(\nu)}(0)}{\nu !}\right| \leq \frac{\left\|f-\varphi_{l}\right\|_{\bar{B}(0,1 / 2)}}{(1 / 2)^{\nu}}
$$

for every $\nu \geq 0$. By using (7), we now estimate

$$
\begin{aligned}
d_{Y}\left(L_{n} f, L_{n} \varphi_{l}\right) & =\sup _{z \in K}\left|\sigma_{\mathcal{A}}(m(n), f)(z)-\sigma_{\mathcal{A}}\left(m(n), \varphi_{l}\right)(z)\right| \\
& =\sup _{z \in K}\left|\sum_{\nu=0}^{\infty} \alpha_{m(n) \nu} s(\nu, f)(z)-\sum_{\nu=0}^{\infty} \alpha_{m(n) \nu} s\left(\nu, \varphi_{l}\right)(z)\right| \\
& =\sup _{z \in K}\left|\sum_{\nu=0}^{\infty} \alpha_{m(n) \nu} s\left(\nu, f-\varphi_{l}\right)(z)\right| \\
& =\sup _{z \in K}\left|\sum_{\nu=0}^{\infty} \alpha_{m(n) \nu} \sum_{i=0}^{\nu} \frac{f^{(i)}(0)-\varphi^{(i)}(0)}{i !} z^{i}\right| \\
& \leq\left\|f-\varphi_{l}\right\|_{\bar{B}(0,1 / 2)} \sum_{\nu=0}^{\infty}\left|\alpha_{m(n) \nu}\right|(\nu+1)\left(2 R_{0}\right)^{\nu} .
\end{aligned}
$$

The last series converges because $\lim _{\nu \rightarrow \infty}\left|\alpha_{n \nu}\right|^{\frac{1}{\nu}}=0$ for all $n \in \mathbb{N}$. Hence we obtain

$$
\lim _{l \rightarrow \infty} d_{Y}\left(L_{n} f, L_{n} \varphi_{l}\right)=0
$$

for every $n \in \mathbb{N}$.

From (6), (8) and the definitions of $d_{X}, d_{Y}$, we derive, exactly as in the proof of Theorem 1.2 in [27], that

$$
\lim _{j \rightarrow \infty} \sup _{n \in \mathbb{N}} \inf _{h \in A}\left\{d_{X}\left(f_{j}, h\right)+d_{Y}\left(L_{n} f_{j}, L_{n} h\right)\right\}=0
$$

for every sequence $\left(f_{j}\right)$ with $f_{j} \in A_{j}$ for all $j \in \mathbb{N}$. According to Theorem 2.1, the sequence $\left(L_{n}\right)$ is densely universal. Thus, by Theorem 2.2, we deduce the residuality of $\mathcal{U}\left(\left.L_{n}\right|_{A}\right)$ in $A$. Hence, we have proved our claim and, consequently, we have (5) at our disposal. 
Thanks to (5) we can pick a function $f_{1} \in B_{1} \cap U(\mathbb{D}, \mathcal{A},(n))$. Therefore, we may select for each $k \in \mathbb{N}$ an strictly increasing sequence $\left(n_{j}^{(1, k)}\right)_{j \geq 1} \subset \mathbb{N}$ such that

$$
\sigma_{\mathcal{A}}\left(\left(n_{j}^{(1, k)}\right), f_{1}\right) \longrightarrow 0 \quad(j \rightarrow \infty) \text { uniformly on } K_{k} \text {. }
$$

For convenience of notation, we set $n_{j}^{(0, k)}:=j$ for all $j, k$. Due to (5) and the fact that a countable intersection of residual subsets is still residual, we may choose a function $f_{2} \in B_{2} \cap \bigcap_{k=1}^{\infty} U\left(\mathbb{D}, \mathcal{A},\left(n_{j}^{(1, k)}\right), K_{k}\right)$. As before, there exists for each $k \in \mathbb{N}$ an strictly increasing sequence $\left(n_{j}^{(2, k)}\right)_{j \geq 1} \subset\left(n_{j}^{(1, k)}\right)_{j \geq 1}$ such that, for every $k$,

$$
\sigma_{\mathcal{A}}\left(\left(n_{j}^{(2, k)}\right), f_{2}\right) \longrightarrow 0(j \rightarrow \infty) \text { uniformly on } K_{k}
$$

Observe that, for each $k$,

$$
\sigma_{\mathcal{A}}\left(\left(n_{j}^{(2, k)}\right), f_{1}\right) \longrightarrow 0(j \rightarrow \infty) \text { uniformly on } K_{k}
$$

By induction, we can select a sequence $\left(f_{N}\right)$ of functions as well as a countably many sequences $\left(n_{j}^{(N, k)}\right)_{j \geq 1}(N, k \in \mathbb{N})$ satisfying

$$
f_{N} \in B_{N} \cap \bigcap_{k=1}^{\infty} U\left(\mathbb{D}, \mathcal{A},\left(n_{j}^{(N-1, k)}\right), K_{k}\right) \quad(N \in \mathbb{N})
$$

and, for every $k \in \mathbb{N}$,

$$
\sigma_{\mathcal{A}}\left(\left(n_{j}^{(N, k)}\right), f_{l}\right) \longrightarrow 0(j \rightarrow \infty) \text { uniformly on } K_{k}(l=1, \ldots, N) .
$$

Let us define

$$
\mathcal{D}:=\operatorname{span}\left\{f_{N}: N \in \mathbb{N}\right\} .
$$

Then $\mathcal{D}$ is, trivially, a linear submanifold of $H(\mathbb{D})$. Moreover, since $f_{N} \in B_{N}$, we have $\left\|f_{N}-P_{N}\right\|_{\bar{B}\left(0, \frac{N}{N+1}\right)}<1 / N \rightarrow 0(N \rightarrow \infty)$. Since $\left(P_{N}\right)$ is a dense sequence in $H(\mathbb{D})$ and $\left(\bar{B}\left(0, \frac{N}{N+1}\right)\right)$ is an exhaustive sequence of compact subsets of $\mathbb{D}$, we derive that the set $\left\{f_{N}: N \in \mathbb{N}\right\}$ is dense too. Consequently, $\mathcal{D}$ is also dense.

Our next goal is to demonstrate that each function $f \in \mathcal{D} \backslash\{0\}$ is $\mathcal{A}$ universal. For such a function, there exists $N \in \mathbb{N}$ and complex scalars $c_{1}, \ldots, c_{N}$ such that $c_{N} \neq 0$ and $f=c_{1} f_{1}+\cdots+c_{N} f_{N}$. Since $U(\mathbb{D}, \mathcal{A},(n))=$ $\bigcap_{k=1}^{\infty} U\left(\mathbb{D}, \mathcal{A},(n), K_{k}\right)$, it must be shown that, for prescribed $k \in \mathbb{N}$ and $h \in$ $A\left(K_{k}\right)$, there exists some sequence $\left(\lambda_{j}\right) \subset \mathbb{N}$ such that $\sigma_{\mathcal{A}}\left(\lambda_{j}, f\right) \rightarrow h$ uniformly on $K_{k}$. For this, observe that because of $f_{N} \in U\left(\mathbb{D}, \mathcal{A},\left(n_{j}^{(N-1, k)}\right)_{j \geq 1}, K_{k}\right)$, there is a subsequence $\left(\lambda_{j}\right)$ of $\left(n_{j}^{(N-1, k)}\right)$ satisfying

$$
\sigma_{\mathcal{A}}\left(\lambda_{j}, f_{N}\right) \longrightarrow c_{N}^{-1} h \quad(j \rightarrow \infty) \text { uniformly on } K_{k} \text {. }
$$


We also have that $\sigma_{\mathcal{A}}\left(\lambda_{j}, f_{l}\right) \rightarrow 0$ uniformly on $K_{k}$ for $l=1, \ldots, N-1$. Consequently

$$
\begin{aligned}
\sigma_{\mathcal{A}}\left(\lambda_{j}, f\right)= & c_{N} \sigma_{\mathcal{A}}\left(\lambda_{j}, f_{N}\right)+\sum_{l=1}^{N-1} c_{l} \sigma_{\mathcal{A}}\left(\lambda_{j}, f_{l}\right) \\
& \longrightarrow h+0=h \quad(j \rightarrow \infty) \text { uniformly on } K_{k},
\end{aligned}
$$

as required.

Finally, fix $n \in \mathbb{N}, \gamma \in \Gamma_{0}(\mathbb{D})$ and $f \in \mathcal{D} \backslash\{0\}$. It remains to prove that $C_{\gamma}\left(T_{n} f\right)=\mathbb{C}_{\infty}$. As before, $f=c_{1} f_{1}+\cdots+c_{N} f_{N}$, where $N \in \mathbb{N}$ and $c_{N} \neq 0$. The only property of $\gamma$ that we will use is that it intersects the compact sets $\widetilde{S}_{j}$ for all sufficiently large $j$. Therefore, there is $l_{0} \in \mathbb{N}$ such that we can select a point

$$
z_{l} \in \gamma \cap \widetilde{S}_{p(n, N, l)} \quad \text { for all } \quad l \geq l_{0}
$$

Since $f_{k} \in B_{k}$, one may assume without loss of generality that $l_{0}$ is such that

$$
\left\|f_{N}-q_{l} h_{n}\right\|_{\widetilde{S}_{p(n, N, l)}}<\delta\left(\frac{1}{l}, n, p(n, N, l)\right)
$$

and

$$
\left\|f_{k}\right\|_{\widetilde{S}_{p(n, N, l)}}<\delta\left(\frac{1}{l}, n, p(n, N, l)\right) \quad(k=1, \ldots, N-1)
$$

for all $l \geq l_{0}$. By using (3), (4), (9), (10) and (11), we obtain for every $l \geq l_{0}$ that

$$
\left|\left(T_{n} f_{N}\right)\left(z_{l}\right)-q_{l}\right|<\frac{1}{l} \quad \text { and } \quad\left|\left(T_{n} f_{k}\right)\left(z_{l}\right)\right|<\frac{1}{l}(k=1, \ldots, N-1) .
$$

Since $T_{n}$ is linear, we get from the triangle inequality that

$$
\begin{aligned}
\left|\left(T_{n} f\right)\left(z_{l}\right)-c_{N} q_{l}\right| & =\left|c_{1}\left(T_{n} f_{1}\right)\left(z_{l}\right)+\cdots+c_{N}\left(T_{n} f_{N}\right)\left(z_{l}\right)-c_{N} q_{l}\right| \\
& \leq\left(\sum_{k=1}^{N}\left|c_{k}\right|\right) \cdot \frac{1}{l} \longrightarrow 0 \quad(l \rightarrow \infty) .
\end{aligned}
$$

But $c_{N} \neq 0$, so the sequence $\left\{c_{N} q_{l}: l \in \mathbb{N}\right\}$ is dense in $\mathbb{C}_{\infty}$. Since $\lim _{l \rightarrow \infty}\left(\left(T_{n} f\right)\left(z_{l}\right)-c_{N} q_{l}\right)=0$, we derive that the set $\left\{\left(T_{n} f\right)\left(z_{l}\right): l \geq l_{0}\right\}$ is also dense in $\mathbb{C}_{\infty}$. Therefore, every value $\alpha \in \mathbb{C}_{\infty}$ belongs to the cluster set $C_{\gamma}\left(T_{n} f\right)$. The proof is finished.

If, specially, $\mathcal{A}$ is the identity matrix and every $T_{n}$ is the identity operator, then one obtains the following corollary, that asserts the announced algebraic genericity of $U(\mathbb{D}) \cap M C S\left(\Gamma_{0}(\mathbb{D})\right)$. 
Corollary 3.2. There exists a dense linear manifold $\mathcal{D} \subset H(\mathbb{D})$ such that every function $f \in \mathcal{D} \backslash\{0\}$ is a universal Taylor series and has maximal cluster set along any curve $\gamma \in \Gamma_{0}(\mathbb{D})$.

\section{Behavior along the radii and other curves}

We start this section by showing the unboundedness of the images of most radii under any universal Taylor series $f$, when $f$ is subjected to the action of a linear differential operator $P(D)$. Here $P$ is a polynomial with complex coefficients.

Theorem 4.1. Let $f$ be a universal Taylor series. Then there is a residual subset $M \subset \mathbb{T}$ such that, for every non-zero polynomial $P$ and every $\zeta \in M$, the set

is unbounded.

$$
\{(P(D) f)(r \zeta): 0<r<1\}
$$

Proof. According to [8, Theorem 3.2], there is a residual subset $M \subset \mathbb{T}$ such that, for every $\zeta \in M$, the set $\{f(r \zeta): 0<r<1\}$ is unbounded. We will prove that $M$ is also valid for any operator $P(D)$, where $P$ is a non-zero polynomial. It is evident that one can assume that the leading coefficient of $P$ is 1 . Hence, if $P$ is a constant, the desired conclusion is exactly [8, Theorem 3.2]. So we may suppose that $P$ has the form $P(z)=$ $\left(z-a_{1}\right) \cdots\left(z-a_{N}\right)$, where $N \in \mathbb{N}$ and $a_{1}, \ldots, a_{N} \in \mathbb{C}$.

Let $f$ be a universal Taylor series, $Q(z):=z-a(a \in \mathbb{C})$ and $g:=Q(D) f$. By elementary integration, we obtain

$$
f(z)=\left(f(0)+\int_{0}^{z} g(t) e^{-a t} d t\right) e^{a z},
$$

where we can take the segment $[0, z]$ as integration curve. Fix $\zeta \in M$ and assume, by way of contradiction, that the set $\{(Q(D) f)(r \zeta): 0<r<1\}$ is bounded. By (12),

$$
|f(r \zeta)| \leq e^{|a|}|f(0)|+e^{2|a|} \sup _{(0, \zeta)}|g|=: \alpha \quad \text { for all } \quad r \in(0,1)
$$

where $\alpha$ is a finite constant. This is absurd, so the set $\{(Q(D) f)(r \zeta): 0<$ $r<1\}$ is unbounded for every $\zeta \in M$. But, since the operator $P(D)$ can be decomposed into finitely many factors of the form $Q(D)$, an induction procedure leads easily to the conclusion.

Next, we present the promised statement about a certain "good" boundary behavior of some universal series (Theorem 4.2). In fact, we have a new generic property, clearly non compatible with that of Theorem 3.1. Observe 
that the decay of the functions along the paths can be made as "tamed" as desired. Except for the first step, its proof follows closely the proofs of our Theorem 3.1 and of Theorem 1.2 in [27], so it will be shortened at some points. In [51, Example 2] it is asserted the existence of a non-zero function $f \in H(\mathbb{D})$ which has at each point $\zeta \in \mathbb{T}$ a path $P_{\zeta}$ which is tangent to the radius joining the origin to $\zeta$ and $\lim _{\substack{z \rightarrow \zeta \\ z \in P_{\zeta}}} f(z)=0$. We state here that this property is compatible with universality.

Theorem 4.2. There is a family of paths $\left\{P_{\zeta}\right\}_{\zeta \in \mathbb{T}}$ with the following properties:

(a) For every $\zeta \in \mathbb{T}, P_{\zeta} \subset \mathbb{D}$ and $P_{\zeta}$ is tangent to the radius joining the origin to $\zeta$.

(b) For each $C$-matrix $\mathcal{A}$ and each continuous function $\alpha: \mathbb{D} \rightarrow(0,+\infty)$, there exists a dense linear manifold $\mathcal{D}$ satisfying the following:

(i) Every $f \in \mathcal{D} \backslash\{0\}$ is $\mathcal{A}$-universal.

(ii) For every $f \in \mathcal{D}, \lim _{\substack{z \rightarrow \zeta \\ z \in P_{\zeta}}} \frac{f(z)}{\alpha(z)}=0$.

Proof. Inspired by [51, Examples 2 and 11], we construct the following "dyadic tree" $V$ inductively. It is the tree which has four branches starting from the origin. We will suggest how to made it in the first quadrant. In the other three ones it is only needed to rotate this construction. As for the first quadrant, draw the circle $C$ with center $1+i$ passing through the points $1, i$. Focus our attention on the part of this circle contained in $\mathbb{D}$. This circle is tangent to both radii $R(0)$ and $R(\pi / 2)$ at the points $1, i$, respectively. We have denoted $R(\theta):=\{r \exp (i \theta): 0 \leq r<1\}$. The tree $V$ begins with the segment joining the origin $z_{0}:=0$ to the point $z_{1}$ that is the intersection of $R(\pi / 4)$ with $C$. The tree is continued by drawing the two branches starting at $z_{1}$ and ending at the points $z_{2,1}:=C \cap R(\pi / 8)$ and $z_{2,2}:=C \cap R(3 \pi / 8)$. From each of these points two new branches are to be constructed. We do it for $z_{2,1}$, the construction being symmetric for $z_{2,2}$. The point $z_{2,1}$ is joined to the points $C \cap R(\pi / 16), C(\pi / 8) \cap R(3 \pi / 16)$, where $C(\theta)$ is the reflection of $C$ by $R(\theta)$ (which is tangent to $R(2 \theta)$ at $\exp (i \pi 2 \theta)$ ). The tree is continued in this way, always by joining each point $P$ in each generation to two new points $Q, S$, so that if $P \in R\left(\nu \pi / 2^{n+1}\right)$ then $Q, S \in R\left((2 \nu \pm 1) \pi / 2^{n+2}\right)$. Each point $Q$ or $S$ is obtained as intersection of the radius containing it with the image of $C$ under reflection (or under a finite sequence of reflections) by an adequate radius $R(\theta)$ (if $P \in C$ then one of the points $Q, S$ is also in $C$ ). If we fix a point $\zeta \in \mathbb{T}$ and consider an adequate sequence of successive branches taken from $V$, then we can construct a path $P_{\zeta} \subset \mathbb{D}$ terminating 
at $\zeta$. The fact that the circles $C$ and $C(\theta)$ are respectively tangent to the radii at $1,1+i$ and at $\exp (i \pi 2 \theta)$ forces the path $P_{\zeta}$ to be tangent at $\zeta$.

Next, fix a $C$-matrix $\mathcal{A}$. Fix also a continuous function $\alpha: \mathbb{D} \rightarrow(0,+\infty)$. By replacing $\alpha$ by the function $\alpha^{*}(z):=\min \{\alpha(w):|w| \leq|z|\}$, one can suppose without loss of generality that $\alpha$ only depends on the modulus $|z|$, i.e. $\alpha(z)=\alpha(|z|)$. Observe that the function $\varepsilon: t \in[0,1) \mapsto(1-t) \alpha(t) \in$ $(0,+\infty)$ is also continuous.

Choose sequences $\left(P_{k}\right)$ and $\left(K_{k}\right)$ as in the proof of Theorem 3.1. Since $\mathbb{D}^{\omega} \backslash V$ is easily seen to be connected and locally connected at $\omega$, and $V$ has no "large islands" near the boundary (because $V^{0}=\emptyset$ ), the set $V$ satisfies the hypotheses of the Nersesjan theorem. And it is easily checked that the sets $V_{k}:=\bar{B}\left(0, \frac{k}{k+1}\right) \cup W_{k}\left(\right.$ where $\left.W_{k}:=V \cap\left\{w:|w| \geq \frac{k+1}{k+2}\right\}, k \in \mathbb{N}\right)$ also satisfy them. Consequently, each set

$B_{k}:=\left\{f \in H(\mathbb{D}):\left\|f-P_{k}\right\|_{\bar{B}\left(0, \frac{k}{k+1}\right)}<\frac{1}{k}\right.$ and $|f(z)|<\varepsilon(|z|)$ for all $\left.z \in W_{k}\right\}$

is nonempty. It is also a $G_{\delta}$ subset of $H(\mathbb{D})$.

Fix $k \in \mathbb{N}$. The set $A:=B_{k}$ can be written as $A=\bigcap_{j=k+1}^{\infty} A_{j}$, where

$$
\begin{aligned}
A_{j}:= & \left\{f \in H(\mathbb{D}):\left\|f-P_{k}\right\|_{\bar{B}\left(0, \frac{k}{k+1}\right)}<1 / k\right. \text { and } \\
& \left.|f(z)|<\varepsilon(|z|) \text { for all } z \in W_{k} \cap \bar{B}\left(0, \frac{j+1}{j+2}\right)\right\} .
\end{aligned}
$$

Now, fix $j \in \mathbb{N}$ with $j \geq k+1$ and $f \in A_{j}$. We want to show the existence of a sequence $\left(\varphi_{l}\right)_{l \geq 1} \subset A$ such that

$$
\lim _{l \rightarrow \infty}\left\|f-\varphi_{l}\right\|_{\bar{B}\left(0, \frac{j+1}{j+2}\right)}=0 .
$$

Similarly to [27, Proof of Lemma 3.2], we consider the set $F:=\bar{B}\left(0, \frac{j+1}{j+2}\right) \cup$ $V=\bar{B}\left(0, \frac{j+1}{j+2}\right) \cup W_{j}$ and the function $T: F \rightarrow \mathbb{C}$ given by

$$
T(z)= \begin{cases}f(z) & \text { if } z \in \bar{B}\left(0, \frac{j+1}{j+2}\right) \\ \frac{\varepsilon(|z|)}{\varepsilon\left(\frac{j+1}{j+2}\right)} f(\pi(z)) & \text { if } z \in W_{j} .\end{cases}
$$

Here $\pi(z)$ denotes the unique point where the circle $\left\{w:|w|=\frac{j+1}{j+2}\right\}$ is intersected by the part of the tree $V$ that, starting from $z$, goes back to the origin. The function $T$ is well defined and $T \in A(F)$. In addition, $F$ satisfies the conditions allowing the application of Nersesjan's theorem. Since $\varepsilon(|\pi(z)|)=\varepsilon\left(\frac{j+1}{j+2}\right)$ for $z \in W_{j}$, we have that $|T(z)|<\varepsilon(|z|)$ on $W_{k}$. 
For every $l=1,2, \ldots$ and every $z \in W_{k}$, set

$$
\delta_{l}(z)=\min \left\{\frac{1}{l}, \frac{1}{k}-\left\|f-P_{k}\right\|_{\bar{B}\left(0, \frac{k}{k+1}\right)}, \varepsilon(|z|)-|T(z)|\right\} .
$$

Since $W_{k}$ is closed in $F$, Tietze's extension theorem permits to extend $\delta_{l}$ to $F$ so that $0<\delta_{l}(z) \leq \min \left\{\frac{1}{l}, \frac{1}{k}-\left\|f-P_{k}\right\|_{\bar{B}\left(0, \frac{k}{k+1}\right)}\right\}$.

By Nersesjan's theorem, we obtain a sequence $\left(\varphi_{l}\right)_{l \geq 1} \subset H(\mathbb{D})$ satisfying

$$
\left|T(z)-\varphi_{l}(z)\right|<\delta_{l}(z) \text { for all } z \in F \text {. }
$$

Then (13) is derived from (14), because $\delta_{l} \leq 1 / l$ and $T=f$ on the ball $\bar{B}\left(0, \frac{j+1}{j+2}\right)$. The fact that each $\varphi_{l} \in A$ follows easily if we distinguish the three cases " $z \in \bar{B}\left(0, \frac{k}{k+1}\right)$ ", " $z \in W_{k} \cap \bar{B}\left(0, \frac{j+1}{j+2}\right)$ ", " $z \in W_{j}$ ", and if we use the definition of $T$ as well as the triangle inequality.

Exactly as in the proof of Theorem 3.1 -that is, by starting from (13) and applying Herzog's theorem (Theorem 2.2)- we can find a sequence $\left(f_{N}\right)$ in $H(\mathbb{C})$ satisfying:

- $f_{N} \in B_{N}$ for every $N$.

- $\left(f_{N}\right)$ is dense in $H(\mathbb{D})$. Hence the linear manifold $\mathcal{D}$ spanned by this sequence is a dense linear submanifold of $H(\mathbb{D})$.

- Each non-zero function $f \in \mathcal{D}$ is $\mathcal{A}$-universal.

Consequently, since $\mathcal{D}$ is a linear manifold, it only remains to prove the property (ii) of the statement of the theorem for each function $f_{k}$. This is easily seen from the fact that $f_{k} \in B_{k}$. Indeed, for $z \in V$ and $|z|$ close enough to 1 , we have $\left|f_{k}(z)\right|<\varepsilon(|z|)=(1-|z|) \alpha(z)$. Hence, for each $\zeta \in \mathbb{T}$, we have $\left|f_{k}(z) / \alpha(z)\right|<1-|z| \rightarrow 0$ as $z \rightarrow \zeta$ along $P_{\zeta}$, because $P_{\zeta} \subset V$. The proof is finished.

We remark that Theorem 4.2 does not contradict the Lusin-Privalov theorem (see [40] and [47, p. 60]), because the limits are not taken along the radii themselves.

\section{Closed linear manifolds of wild functions}

In this final section, we establish the spaceability of the set $U(\mathbb{D}) \cap$ $M C S\left(\Gamma_{0}(\mathbb{D})\right)$ in $H(\mathbb{D})$. For this, we will follow closely the proof of Theorem 2 in [7], so some arguments will be simplified. We need the following refinement of Mergelyan's theorem, that is essentially proved in [7].

Lemma 5.1. Let $\varepsilon>0, N \in \mathbb{N}, r \in(0,1), K \in \mathcal{M}(\mathbb{C} \backslash r \mathbb{D}), L \in \mathcal{M}(r \mathbb{D})$, and $g \in A(K)$. Then there exists a polynomial $P(z)=\sum_{n=N}^{q} a_{n} z^{n}$ such that

$$
\|P\|_{L}<\varepsilon \quad \text { and }\|P-g\|_{K}<\varepsilon .
$$


Theorem 5.2. There exists a closed infinite dimensional linear manifold $\mathcal{F} \subset H(\mathbb{D})$ such that every function $f \in \mathcal{F} \backslash\{0\}$ is a universal Taylor series and has maximal cluster set along any curve $\gamma \in \Gamma_{0}(\mathbb{D})$.

Proof. Choose a dense sequence $\left\{q_{k}\right\}_{k \geq 1}$ in $\mathbb{C}$. As in the proof of $[7$, Theorem 2], fix a sequence $\left\{K_{m}\right\}_{m \geq 1} \subset \mathcal{M}(\mathbb{C} \backslash \mathbb{D})$ such that, for every set $K \in \mathcal{M}(\mathbb{C} \backslash \mathbb{D})$, there exists an $m \in \mathbb{N}$ with $K \subset K_{m}[46]$. Let $\left\{Q_{l}\right\}_{l \geq 1}$ be an enumeration of all polynomials with coefficients in $\mathbb{Q}+i \mathbb{Q}$ and let $\varphi, \psi: \mathbb{N} \rightarrow \mathbb{N}$ be two functions such that, given any couple $(m, l)$ in $\mathbb{N} \times \mathbb{N}$, there exist infinitely many $j$ with $(\varphi(j), \psi(j))=(m, l)$. Fix also a pair of double sequences $\left\{r_{i, j}\right\}_{i \geq j}$ and $\left\{s_{i, j}\right\}_{i \geq j}$ with $\frac{1}{2}<r_{1,1}<s_{1,1}<\frac{2}{3}<r_{2,1}<$ $s_{2,1}<\frac{3}{4}<r_{2,2}<s_{2,2}<\frac{4}{5}<r_{3,1}<s_{3,1}<\frac{5}{6}<r_{3,2}<s_{3,2}<\frac{6}{7}<r_{3,3}<s_{3,3}<$ $\frac{7}{8}<\cdots \rightarrow 1$, and consider the spiral-like compact sets

$$
S_{i, j}=\left\{\left(r_{i, j}+\frac{\left(s_{i, j}-r_{i, j}\right) \theta}{4 \pi}\right) \exp (i \theta): \theta \in[0,4 \pi]\right\} \quad(i, j \in \mathbb{N}, i \geq j) .
$$

Observe that the sets $S_{i, j}$ are contained in $\mathbb{D}$ and are pairwise disjoint.

We use induction to build sequences of polynomials $\left\{f_{j, k}\right\}_{j \geq k}$ for $k \in \mathbb{N}$. Let $\left\{\tau_{n}\right\}_{n \geq 1} \subset(0,+\infty)$ be a sequence such that $\sum_{n=1}^{\infty} \tau_{n}<1$.

First, we define $g_{1,1}=2 z+P(z)$, where $P$ is given by Lemma 5.1 with $K=K_{\varphi(1)} \cup S_{1,1}, L=\bar{B}\left(0, \frac{1}{2}\right), N=2, \varepsilon=\frac{\tau_{1}}{2^{3}}$ and

$$
g(z)= \begin{cases}Q_{\psi(1)}(z)-2 z & \text { if } z \in K_{\varphi(1)} \\ q_{1}-2 z & \text { if } z \in S_{1,1}\end{cases}
$$

The Taylor series of $g_{1,1}$ approaches $Q_{\psi(1)}$ on $K_{\varphi(1)}$. We now correct its value on $K_{\varphi(2)}$ for further expansions by setting $f_{1,1}=g_{1,1}+Q(z)$, where $Q$ is given by Lemma 5.1 with $K=K_{\varphi(2)}, L=\bar{B}\left(0, \frac{2}{3}\right), N=\operatorname{degree}(P)+1$, $\varepsilon=\frac{\tau_{1}}{2^{3}}$ and $g=-g_{1,1}$.

Let us introduce the notation $A(j):=\left\{z: \frac{j}{j+1}<|z|<\frac{j+1}{j+2}\right\}(j \in \mathbb{N})$. Assume that polynomials $f_{j-1, k}$ have been built in the previous step for $k \leq j-1$. Let $N_{j, 1}=1+\max _{1 \leq k \leq j-1} \operatorname{degree}\left(f_{j-1, k}\right)$. We define a intermediate polynomial $g_{j, 1}=f_{j-1,1}+P(z)$, where $P$ is given by Lemma 5.1 with $K=$ $K_{\varphi(j)} \cup S_{k, i}$ [where $(k, i)$ is the unique pair $(k(j), i(j))$ such that $S_{k, i} \subset A(j)$ ], $L=\bar{B}\left(0, \frac{j}{j+1}\right), N=N_{j, 1}, \varepsilon=\frac{\tau_{1}}{2^{j+2}}$ and $g: K \rightarrow \mathbb{C}$ the function defined by

$$
g(z)= \begin{cases}Q_{\psi(j)}(z)-f_{j-1,1}(z) & \text { if } z \in K_{\varphi(j)} \\ q_{k}-f_{j-1,1}(z) & \text { if } z \in S_{k, i} \text { and } i=1 \\ -f_{j-1,1}(z) & \text { if } z \in S_{k, i} \text { and } i \neq 1\end{cases}
$$

Now we correct the Taylor series of $g_{j, 1}$ by setting

$$
f_{j, 1}=g_{j, 1}+Q(z)
$$


where $Q$ is given by Lemma 5.1 with $K=K_{\varphi(j+1)}, L=\bar{B}\left(0, \frac{j+1}{j+2}\right), N=$ degree $(P)+1, \varepsilon=\frac{\tau_{1}}{2^{j+2}}$ and $g=-g_{j, 1}$.

Consequently, the following inequalities are satisfied:

$$
\begin{aligned}
& \left\|f_{j, 1}-f_{j-1,1}\right\|_{\bar{B}\left(0, \frac{j}{j+1}\right)} \leq \frac{\tau_{1}}{2^{j+1}} \\
& \left\|f_{j, 1}\right\|_{K_{\varphi(j+1)}} \leq \frac{\tau_{1}}{2^{j+1}} \\
& \left\|f_{j, 1}-q_{k}\right\|_{S_{k, 1}} \leq \frac{\tau_{1}}{2^{j+1}} \text { if } S_{k, 1} \subset A(j) \\
& \left\|f_{j, 1}\right\|_{S_{k, i}} \leq \frac{\tau_{1}}{2^{j+1}} \text { if } i \neq 1 \text { and } S_{k, i} \subset A(j)
\end{aligned}
$$

Taking $N_{j, 2}=\operatorname{degree}\left(f_{j, 1}\right)+1$, we apply the same construction to deduce $f_{j, 2}$ from $f_{j-1,2}$, and inductively we build polynomials $f_{j, k}(1 \leq k \leq j-1)$ satisfying inequalities similar to those of above. Finally, if $N_{j, j}$ is an integer greater than the degree of all polynomials $f_{j, k}(1 \leq k \leq j-1)$, then $f_{j, j}$ is deduced from $2^{N_{j, j}} z^{N_{j, j}}$ by following the same process.

The condition (15) ensures that the sequence $\left\{f_{j, k}\right\}_{j \geq k}$ converges uniformly on any compact subset of $\mathbb{D}$ to a function $f_{k} \in H(\mathbb{D})$. Let $E$ be the vector space consisting of all series $\sum_{k=1}^{\infty} c_{k} f_{k}$ converging uniformly on compacta of $\mathbb{D}$, and let $\mathcal{F}$ be the closure of $E$ in $H(\mathbb{D})$. By using estimations (15)-(16), it can be proved as in [7] that $\left\{f_{k}\right\}_{k \geq 1}$ is a basic sequence in $L^{2}\left(\frac{1}{2} \mathbb{T}\right)$ [= the space of measurable complex functions $f$ : $\mathbb{T} \rightarrow \mathbb{C}$ with square-integrable modulus, endowed with the norm $\|f\|_{2}:=$ $\left.\left(\int_{0}^{2 \pi}\left|f\left(\frac{1}{2} e^{i \theta}\right)\right|^{2} \frac{d \theta}{2 \pi}\right)^{1 / 2}\right]$ which is equivalent to $\left\{(2 z)^{k}\right\}_{k \geq 1}$, and that $\mathcal{F}$ is a closed infinite dimensional linear manifold such that all $\mathcal{F} \backslash\{0\} \subset U(\mathbb{D})$. Note that convergence in $H(\mathbb{D})$ implies quadratic convergence in the space $L^{2}\left(\frac{1}{2} \mathbb{T}\right)$.

Let us fix a function $f \in \mathcal{F} \backslash\{0\}$ and a curve $\gamma \in \Gamma_{0}(\mathbb{D})$. Note that $\gamma$ intersects all spirals $S_{k, i}$ except finitely many of them. It remains to show that $C_{\gamma}(f)=\mathbb{C}_{\infty}$. Let $\sum_{n=1}^{\infty} c_{n} f_{n}$ be its representation on $L^{2}\left(\frac{1}{2} \mathbb{T}\right)$ (perhaps not convergent in $H(\mathbb{D})$ ). Since $\mathcal{F}$ and $M C S\left(\Gamma_{0}(\mathbb{D})\right)$ are invariant under scaling, one can assume that there exists $N \in \mathbb{N}$ such that $c_{N}=1$. Let $\left\{h_{l}:=\sum_{n=0}^{\infty} c_{n, l} f_{n}\right\}_{l \geq 1}$ be a sequence of series of $E$ converging compactly to $f$. By the continuity of the projections, one can assume that $c_{N, l}=1$ for all $l \in \mathbb{N}$. Due to the density of $\left(q_{k}\right)$ in $\mathbb{C}$ and to the facts that sets $S_{k, N}$ tend to the boundary $\mathbb{T}$ as $k \rightarrow \infty$ and $\gamma$ intersects almost all of them, it is enough to demonstrate that

$$
\lim _{k \rightarrow \infty}\left\|f-q_{k}\right\|_{S_{k, N}}=0 .
$$

Fix $k \in \mathbb{N}$ with $k \geq N$ and let $j=j(k)$ the unique positive integer with $S_{k, N} \subset A(j)$. Observe that $j(k) \rightarrow \infty$ as $k \rightarrow \infty$. 
Let us choose $l$ such that $\left\|f-h_{l}\right\|_{S_{k, N}}<1 / k$. Since convergence in $H(\mathbb{D})$ is stronger than convergence in $L^{2}\left(\frac{1}{2} \mathbb{T}\right)$, we have that $\left(h_{l}\right)$ converges in $L^{2}\left(\frac{1}{2} \mathbb{T}\right)$, so the sequence $\left(\left\|h_{l}\right\|_{2}\right)$ is bounded, say $\left\|h_{l}\right\|_{2} \leq \beta(l \in \mathbb{N})$. From the equivalence of the basic sequences $\left(f_{n}\right)$ and $\left(2^{n} z^{n}\right)$, there is a constant $m \in(0,+\infty)$ such that $m\left\|\sum_{n=1}^{\infty} c_{n, l}(2 z)^{n}\right\|_{2} \leq\left\|\sum_{n=1}^{\infty} c_{n, l} f_{n}\right\|_{2}$ for all $l \geq 1$ (see $[9$, p. 108]). Hence, if we set $\alpha:=\beta / m$, then we obtain

$$
\left(\sum_{n=1}^{\infty}\left|c_{n, l}\right|^{2}\right)^{1 / 2} \leq \alpha \quad(l \in \mathbb{N})
$$

We will employ the inequalities (15), (17), (18) (or their analogues for higher steps) and the fact $S_{k, N} \subset A(j) \subset \bar{B}\left(0, \frac{j+1}{j+2}\right)$. For $n \neq N$, we get

$$
\begin{aligned}
\left\|f_{n}\right\|_{S_{k, N}} & \leq\left\|f_{j, n}\right\|_{S_{k, N}}+\left\|f_{n}-f_{j, n}\right\|_{S_{k, N}} \\
& \leq\left\|f_{j, n}\right\|_{S_{k, N}}+\sum_{p=j+1}^{\infty}\left\|f_{p, n}-f_{p-1, n}\right\|_{\bar{B}\left(0, \frac{p}{p+1}\right)} \\
& \leq \frac{\tau_{n}}{2^{j+1}}+\sum_{p=j+1}^{\infty} \frac{\tau_{n}}{2^{p+1}}=\frac{\tau_{n}}{2^{j}}
\end{aligned}
$$

On the other hand, we have, analogously,

$$
\begin{aligned}
\left\|f_{N}-q_{k}\right\|_{S_{k, N}} & \leq\left\|f_{j, N}-q_{k}\right\|_{S_{k, N}}+\left\|f_{N}-f_{j, N}\right\|_{S_{k, N}} \\
& \leq\left\|f_{j, N}-q_{k}\right\|_{S_{k, N}}+\sum_{p=j+1}^{\infty}\left\|f_{p, N}-f_{p-1, N}\right\|_{\bar{B}\left(0, \frac{p}{p+1}\right)} \\
& \leq \frac{\tau_{N}}{2^{j+1}}+\sum_{p=j+1}^{\infty} \frac{\tau_{N}}{2^{p+1}}=\frac{\tau_{N}}{2^{j}}
\end{aligned}
$$

Consequently, thanks to (20)-(22) and the Cauchy-Schwarz inequality we arrive to

$$
\begin{aligned}
\left\|f-q_{k}\right\|_{S_{k, N}} & \leq\left\|f-h_{l}\right\|_{S_{k, N}}+\left\|h_{l}-q_{k}\right\|_{S_{k, N}} \\
& \leq \frac{1}{k}+\left\|f_{N}-q_{k}\right\|_{S_{k, N}}+\sum_{n \neq N}\left|c_{n, l}\right|\left\|f_{n}\right\|_{S_{k, N}} \\
& \leq \frac{1}{k}+\frac{\tau_{N}}{2^{j}}+\left(\sum_{n=1}^{\infty}\left|c_{n, l}\right|^{2}\right)^{1 / 2}\left(\sum_{n=1}^{\infty} \frac{\tau_{n}^{2}}{4^{j}}\right)^{1 / 2} \\
& \leq \frac{1}{k}+\frac{1}{2^{j(k)}}+\frac{\alpha}{2^{j(k)}} \longrightarrow 0 \quad(k \rightarrow \infty),
\end{aligned}
$$

which proves (19), as desired.

A question that is related to the result in this section is the following. In many instances the existence of closed infinite dimensional subspaces of universal functions depends on the assumption of some Universality Crite- 
rion (see for instance [32]). We want to conclude this paper by posing the problem of finding universality criteria similar to the one established in [19], or the generalization in [16] and [10], as a tool to show the spaceability of the universalities related to the one studied here.

Acknowledgement. We are indebted to the referee for useful suggestions, concerning lineability and recent relevant literature.

\section{References}

[1] Aizpuru, A., Pérez-Eslava, C. and Seonne-Sepúlveda, J. B.: Linear structure of sets of divergent sequences and series. Linear Algebra Appl. 418 (2006), no. 2-3, 595-598.

[2] Armitage, D. H. And Costakis, G.: Boundary behavior of universal Taylor series and their derivatives. Constr. Approx. 24 (2006), no. 1, 1-15.

[3] Aron, R., Bès, J., León, F. And Peris, A.: Operators with common hypercyclic subspaces. J. Operator Theory 54 (2005), no. 2, 251-260.

[4] Aron, R., García, D. and Maestre, M.: Linearity in non-linear problems. Rev. R. Acad. Cienc. Ser. A Mat. 95 (2001), no. 1, 7-12.

[5] Aron, R., Gurariy, V. I. and Seonne, J. B.: Lineability and spaceability of sets of functions on $\mathbb{R}$. Proc. Amer. Math. Soc. 133 (2005), no. 3, 795-803.

[6] BAYART, F.: Topological and algebraic genericity of divergence and universality. Studia Math. 167 (2005), no. 2, 161-181.

[7] BAyART, F.: Linearity of sets of strange functions. Michigan Math. J. 53 (2005), no. 2, 291-303.

[8] Bayart, F.: Boundary behavior and Cèsaro means of universal Taylor series. Rev. Mat. Complut. 19 (2006), no. 1, 235-247.

[9] Beauzamy, B.: Introduction to Banach spaces and their geometry. North Holland Mathematics Studies 68. North Holland Publishing Co., Amsterdam-New York, 1982.

[10] Bermúdez, T. Bonilla, A. And Peris, A.: On hypercyclicity and supercyclicity criteria. Bull. Austral. Math. Soc. 70 (2004), no. 1, 45-54.

[11] Bernal-González, L.: Hypercyclic subspaces in Fréchet spaces. Proc. Amer. Math. Soc. 134 (2006), no. 7, 1955-1961.

[12] Bernal-González, L., Bonilla, A., Calderón-Moreno, M. C. and Prado-Bassas, J. A.: Maximal cluster sets of $L$-analytic functions along arbitrary curves. Constr. Approx. 25 (2007), no. 2, 211-219.

[13] Bernal-González, L., Calderón-Moreno, M. C. and Luh, W.: Universal matrix transforms of holomorphic functions. Houston J. Math. 32 (2006), no. 1, 315-324. 
[14] Bernal-González, A., Calderón-Moreno, M. C. And PradoBassas, J. A.: Maximal cluster sets along arbitrary curves. J. Approx. Theory 129 (2004), no. 2, 207-216.

[15] Bernal-González, A., Calderón-Moreno, M. C. and PradoBassas, J.A.: Simultaneously maximal radial cluster sets. J. Approx. Theory 135 (2005), no. 1, 114-124.

[16] Bernal-González, L. and Grosse-Erdmann, K. G.: The hypercyclicity criterion for sequences of operators. Studia Math. 157 (2003), no. 1, $17-32$.

[17] Bernal-González, L. And Montes-Rodríguez, A.: Non-finite dimensional closed vector spaces of universal functions for composition operators. J. Approx. Theory 82 (1995), no. 3, 375-391.

[18] BÈs, J. P.: Invariant manifolds of hypercyclic vectors for the real scalar case. Proc. Amer. Math. Soc. 127 (1999), no. 6, 1801-1804.

[19] Bès, J. P. And Peris, A.: Hereditarily hypercyclic operators. J. Funct. Anal. 167 (1999), no. 1, 94-112.

[20] Bonet, J., Martínez-Giménez, F. And Peris, A.: Universal and chaotic multipliers on spaces of operators. J. Math. Anal. Appl. 297 (2004), no. 2, 599-611.

[21] BoAs, R. P.: Entire functions. Academic Press Inc., New York, 1954.

[22] Bourdon, P.: Invariant manifolds of hypercyclic vectors. Proc. Amer. Math. Soc. 118 (1993), no. 3, 845-847.

[23] Chan, K. C.: Hypercyclicity of the operator algebra for a separable Hilbert space. J. Operator Theory 42 (1999), no. 2, 231-244.

[24] Chan, K. C. and Taylor, R. D.: Hypercyclic subspaces of a Banach space. Integral Equations Operator Theory 41 (2001), no. 4, 381-388.

[25] Collingwood, E. F. And Lohwater, A. J.: The theory of cluster sets. Cambridge Tracts in Mathematics and Mathematical Physics 56. Cambridge University Press, Cambridge, 1966.

[26] Chui, C. and Parnes, M. N.: Approximation by overconvergence of a power series. J. Math. Anal. Appl. 36 (1971), 693-696.

[27] Costakis, G.: On the radial behavior of universal Taylor series. Monatsh. Math. 145 (2005), no. 1, 11-17.

[28] GaIer, D.: Lectures on Complex Approximation. London Math. Society Lecture Notes 221. Cambridge Univ. Press, 1995.

[29] García-Pacheco, F. J., Palmberg, N. and Seonne-Sepúlveda, J. B.: Lineability and algebrability of pathological phenomena in analysis. J. Math. Anal. Appl. 326 (2007), no. 2, 929-939.

[30] García-Pacheco, F. J. and Seonne-Sepúlveda, J. B.: Vector spaces of non-measurable functions. Acta Math. Sin. (Engl. Ser.) 22 (2006), no. 6, $1805-1808$. 
[31] González, M., León-SaAvedra, F. And Montes-Rodríguez, A.: Semi-Fredholm Theory: hypercyclic and supercyclic subspaces. Proc. London Math. Soc. (3) 81 (2000), no. 1, 169-189.

[32] Grosse-Erdmann, K. G.: Universal families and hypercyclic operators. Bull. Amer. Math. Soc. (N.S.) 36 (1999), no. 3, 345-381.

[33] Gurariy, V. And Quarta, L.: On lineability of sets of continuous functions. J. Math. Anal. Appl. 294 (2004), no. 1, 62-72.

[34] Herzog, G.: On zero-free universal entire functions. Arch. Math. 63 (1994), no. 4, 329-332.

[35] Kahane, J. P.: Baire's category theorem and trigonometric series. J. Anal. Math. 80 (2000), 143-182.

[36] Kierst, St. And Szpilrajn, D.: Sur certaines singularités des fonctions analytiques uniformes. Fund. Math. 21 (1933), 276-294.

[37] Kolyada, S. And Snoha, L.: Some aspects of topological - A survey. In Iteration Theory (ECIT 94) (Opava), 3-35. Grazer Math. Ber. 334. KarlFranzens-Univ. Graz, Graz, 1997.

[38] León-SaAvedra, F. and Montes-Rodríguez, A.: Spectral theory and hypercyclic subspaces. Trans. Amer. Math. Soc. 353 (2001), no. 1, 247-267.

[39] LuH, W.: Approximation analytischer Funktionen durch überkonvergente Potenzreihen und deren Matrix-Transformierten. Mitt. Math. Sem. Giessen Heft 88, 1970.

[40] Lusin, N. And Privalov, J.: Sur l'unicité et la multiplicité des fonctions analitiques. Ann. Sci. École Norm. Sup. 42 (1925), 143-191.

[41] Martínez-Giménez, F. And Peris, A.: Universality and chaos for tensor products of operators. J. Approx. Theory 124 (2003), no. 1, 7-24.

[42] Melas, A. And Nestoridis, V.: Universality of Taylor series as a generic property of holomorphic functions. Adv. Math. 157 (2001), no. 2, 138-176.

[43] Montes-Rodríguez, A.: Banach spaces of hypercyclic vectors. Michigan Math. J. 43 (1996), no. 3, 419-436.

[44] Montes-Rodríguez, A. And Romero-Moreno, M. C.: Supercyclicity in the operator algebra. Studia Math. 150 (2002), no. 3, 201-213.

[45] Nersesjan, A. A.: Carleman sets. Izv. Akad. Nauk Armjan. SSR Ser. Mat. 6 (1971), no. 6, 465-471. English transl. in: Amer. Math. Soc. Transl. (2) 122 (1984), 99-104.

[46] Nestoridis, V.: Universal Taylor series. Ann. Inst. Fourier (Grenoble) 46 (1996), no. 5, 1293-1306.

[47] Noshiro, K.: Cluster sets. Ergebnisse der Mathematik und ihrer Grenzgebiete. N. F., Heft 28. Springer-Verlag, Berlin, 1960.

[48] Oxтoвy, J. C.: Measure and Category. Graduate Texts in Mathematics 2. Springer-Verlag, New York-Berlin, 1980.

[49] Petersson, H.: Hypercyclic subspaces for Fréchet spaces operators. J. Math. Anal. Appl. 319 (2006), no. 2, 764-782. 
[50] READ, C. J.: The invariant subspace problem for a class of Banach spaces, II: Hypercyclic operators. Israel J. Math. 63 (1988), no. 1, 1-40.

[51] Schneider, W. J.: Approximation and harmonic measure. In Aspects of contemporary complex analysis (Proc. NATO Adv. Study Inst., Univ. Durham, Durham, 1979) 333-349. Academic Press, London-New York, 1980

Recibido: 21 de febrero de 2007

Revisado: 19 de febrero de 2008

L. Bernal-González

Departamento de Análisis Matemático Facultad de Matemáticas, Apdo. 1160

Avda. Reina Mercedes 41080 Sevilla, Spain lbernal@us.es

A. Bonilla

Departamento de Análisis Matemático

Universidad de La Laguna C/Astrofísico Fco. Sánchez, s/n 38271 La Laguna, Tenerife, Spain abonilla@ull.es

M.C. Calderón-Moreno

Departamento de Análisis Matemático Facultad de Matemáticas, Apdo. 1160 Avda. Reina Mercedes 41080 Sevilla, Spain mccm@us.es

J.A. Prado-Bassas Departamento de Análisis Matemático Facultad de Matemáticas, Apdo. 1160 Avda. Reina Mercedes 41080 Sevilla, Spain

bassas@us.es

The first, third and fourth authors have been partially supported by the Plan Andaluz de Investigación de la Junta de Andalucía FQM-127 and by MCYT Grant MTM200613997-C02-01. The second author has been partially supported by MEC and FEDER MTM2005-07347. The four authors have been partially supported by MEC (Acción Especial) MTM2006-26627-E. 